\section{Parasitismo por Ascaris lumbricoides em crianças menores de dois anos: estudo populacional em comunidade do Estado do Rio de Janeiro}

\author{
Ascaris lumbricoides in infants: \\ a population-based study in Rio de J aneiro, Brazil
}

Lêda Maria da Costa-Macedo 1

Maria do Carmo Esteves da Costa 2

Liz Maria de Almeida 2

\footnotetext{
1 Departamento de Patologia, Universidade do Estado do Rio de Janeiro. Rua Teodoro da Silva 48, 5 o andar, Rio de Janeiro, RJ 20551-001, Brasil.

2 Núcleo de Estudos de Saúde Coletiva, Universidade Federal do Rio de Janeiro. Av. Brigadei ro Trompowsky s/no, Hospital Universitário, 5o andar, Ala Sul, Il ha do Fundão, Rio de Janeiro, RJ 21941-590, Brasil.
}

Abstract Prevalence and intensity of Ascaris lumbricoides infection were analyzed by a crosssectional study in children addressed in the city of Duque de Caxias, Ri o de Janei ro State, from July to December 1997. The study population consisted of children under two years of age $(n=387)$ and mothers of children under one year of age $(n=104)$. Coproparasitologic tests were run using the MIFC and the Kato thick smear (Kato-Katz) methods. Prevalence was 3.3\% (95\% Cl: 1.0-7.8) for children under one year, $30.7 \%(95 \% \mathrm{Cl}: 25.4-36.4)$ for one-year-olds, and $42.3 \%(95 \% \mathrm{Cl}$ : 33.0-51.9) for mothers of children under one. Some $38.0 \%$ of children under one year of age showed medium-to-heavy infection. These results indicated that ascariasis was an important problem in the first year of life and demonstrated the need for research in the area of maternal and infantileascariasis.

Key words Parasites; Ascaris lumbricoides; Infants; Cross-Sectional Studies; Epidemiology

Resumo A prevalência ea intensidade da infecção por Ascaris lumbricoides foram analisadas mediante um estudo transversal em crianças resi dentes no Município de Duque de Caxias, no Estado do Rio de Janei ro, entre jul ho e dezembro de 1997. A população do estudo foi constituída por crianças com menos de dois anos de idade e as mães das crianças menores de um ano. As amostras fecais foram recol hi das nos domicílios em frascos contendo solução conservadora de MIF e processadas por mei o das técnicas de MIFC e Kato-Katz. As prevalências de infecção por A. lumbricoides observadas foram 3,3\% (I.C. 95\% 1,0 - 7,8) para os menores de um ano, 30,7\% (I.C. 95\% 25,4 - 36,4) para as crianças com um ano de idade e 42,3\% (I.C. 95\% 33,0 - 51,9) para as mães. Infecção com carga parasitária moderada/pesada foi observada em cerca de 38\% das crianças com um ano de idade e $36,0 \%$ das mães. Estes resultados demonstram a importância da ascari ose já no primeiro ano de vida eindi cam a necessidade de aprofundamento na investigação desta parasitose na população materno-infantil.

Palavras-chave Parasitos; Ascaris lumbricoides; Lactentes; Estudos Transversais; Epidemi ologia 
Introdução

Ascarislumbricoidesé o parasito intestinal mais freqüente no mundo. Em estudo para avaliar a prevalência deste enteroparasito, Silva et al. (1997a) estimou que a infecção estaria presente em cerca de $31,0 \%$ dos indivíduos procedentes de regiões endêmicas do mundo. Neste estudo, o Brasil inseriu-se no grupo dos locais mais parasitados, sendo a infecção detectada em cerca de $39,0 \%$ da população. Apesar do crescimento em números absolutos dos indivíduos parasitados em função do aumento geral da população nos últimos 20 anos (IBGE, 1997), verifica-se que o país tem apresentado, em algumas áreas, um decréscimo na prevalência da infecção entre os escolares (Vinha, 1971; Chieffi et al., 1988; Gross et al., 1989). Para o Município do Rio de Janeiro, os dados mais atuais relacionados à prevalência da ascariose em pré-escolares mostram que ela é responsável por cerca de $25,0 \%$ da infecção parasitária neste grupo populacional (Costa-Macedo et al., 1998).

Com relação à criança menor de dois anos, apesar do reduzido número de publicações sobre o assunto neste grupo etário, tem sido relatada a ocorrência do parasitismo em crianças já nos primeiros meses de vida (Costa-Macedo $\&$ Rey, 1997). Alguns estudos para avaliar a prevalência da ascariose, nesta faixa etária, mostram aumento significativo da infecção por A. lumbricoi des entre as menores de um ano em relação às de um ano (Frenzel et al., 1979; Monteiro et al., 1988; Costa-Macedo et al., 1998).

Alguns modelos construídos para avaliar os possíveis fatores relacionados à infecção por helmintos em crianças têm procurado identificar a relação entre o parasitismo nos demais membros das famílias e a ocorrência do parasitismo precoce (Carlier \& Truyens, 1995; Chan et al., 1994).

A intensidade da infecção na população infectada por A. lumbricoides tem sido definida segundo a determinação da carga parasitária, utilizando-se métodos que permitam a contagem de ovos no material examinado. Pesquisando parasitoses intestinais em população favelizada do Município de São Paulo, Ferreira et al. (1991) encontraram, para este helminto, infecção leve (considerada pelos autores quando a carga parasitária era menor que 10000 por grama de fezes) em todas as crianças menores de cinco anos e nos adultos acima de 26 anos de idade. Para menores de dois anos, entretanto, a intensidade da infecção por esse helminto tem sido pouco avaliada em inquéritos epidemiológicos populacionais.
Esse trabalho teve como objetivo verificar a prevalência e a intensidade da infecção por A. lumbricoides em crianças menores de dois anos de idade, residentes em uma comunidade da região metropolitana do Rio de Janeiro. Procuramos avaliar também o parasitismo materno como outro possível indicador das condições sócio-ambientais da comunidade.

\section{Material e métodos}

O inquérito coproparasitológico que originou a base de dados utilizada neste trabal ho foi realizado de julho a dezembro de 1997, no distrito de Campos Elyseos, município de Duque de Caxias. Utilizou-se o desenho de estudo transversal para estimar a prevalência de enteroparasitoses e a carga parasitária em crianças entre 1 e 9 anos de idade. $O$ delineamento deste estudo encontra-se descrito em outro trabalho (Luiz et al., 1997). Paralelamente, foi composta uma sub-amostra de todas as crianças menores de um ano de idade e suas respectivas mães, identificadas no censo realizado pela equipe de pesquisa em 10 setores censitários da área onde se realizou o estudo principal (20 setores censitários).

Foram entregues 231 potes para crianças menores de 12 meses e respectivas mães e foram examinadas 120 destas crianças e 104 mães. Para as crianças de 12 a 23 meses foram entregues 362 frascos, sendo examinadas 267 amostras. A amostra total examinada correspondeu a 491 exames.

Os potes contendo solução conservadora (MIF) foram entregues e recolhidos no local de moradia das pessoas, após orientação do responsável quanto à coleta do material das crianças. As mães das crianças menores de um ano também receberam potes com MIF, sendo orientadas para coleta de seu material. As mães foram orientadas para que coletassem três amostras de fezes (em três dias diferentes) e as armazenassem em um mesmo frasco.

Os exames foram realizados na Disciplina de Parasitologia do Departamento de Patologia e Laboratórios, da Faculdade de Ciências Médicas da Universidade do Estado do Rio de Janeiro. A técnica escol hida para analisar o material fecal foi o de Blagg et al. (1955), com as modificações sugeridas por Coutinho (1956). Além deste método, optou-se pela inclusão do método de Kato-Katz (Katz et al., 1972), específico para ovos de helmintos, que permite a quantificação da carga parasitária da espécie encontrada, e que foi realizada com base no material fixado no MIF. Para a realização do 
método de Kato-Katz foi utilizada a parte mais sólida do material homogeneizado no MIF, seguindo a descrição da técnica para o material colhido à fresco.

A classificação da intensidade de infecção por A. lumbricoides foi baseada nos critérios recomendados pela Organização Mundial da Saúde (WHO, 1987) segundo os quais a infecção é atribuída como leve, quando a carga parasitária encontrada for menor que 5.000 ovos por grama de fezes, moderada quando a carga estiver entre 5.000 e 50.000, e pesada quando ultrapassar 50.000 ovos/grama de fezes.

Os dados foram armazenados e analisados no software Epi Info 6.0. Foram estimadas as médias e respectivos desvios padrão (DP), medianas e prevalências por idade para as crianças e as mães, e determinados os intervalos de $5 \%$ de confiança. Os testes utilizados para comparação foram as estatísticas não paramétricas do $\chi^{2}$ (Pearson).

\section{Resultados}

Foram realizados um total de 491 exames, sendo 387 de crianças menores de 24 meses e 104 de mães de crianças menores de um ano.

A mediana das idades entre as crianças foi 16 meses com primeiro quartil em 8 meses e terceiro quartil em 19 meses. Para o grupo de mães a mediana da idade foi 26 anos, com primeiro quartil em 21 anos e terceiro quartil em 32 anos.

Nos três grupos pesquisados, a infecção por A. I umbricoides correspondeu a pelo menos 2/3 das enteroparasitoses encontradas. As crianças de 12 a 23 meses apresentaram proporções nove vezes maiores de infecção do que as menores de 12 meses. A positividade para A. lumbricoides foi de 3,3 (I.C.95\% 1,0-7,8) para os menores de 12 meses, 30, 7 (I.C. 95\% 25,4-36,6) para os de 12 - 23 meses e 42,3\% (I.C. 95\% 33,0$51,9)$ para as mães. A prevalência total das enteroparasitoses e da ascariose, pelas duas técnicas, encontra-se na Figura 1.

A distribuição das crianças por grupos etários (idade em meses) e segundo o estado de infecção por A. lumbri coides mostrou diferenças estatisticamente significativas na composição etária dos dois subgrupos de infectados e não infectados por A. Iumbricoides ( $p<0,001$ ), sendo observado um predomínio de ascariose entre as crianças maiores. Os valores estimados mostram um aumento das prevalências específicas com a idade (Tabela 1 ).

Na população materna, a maior proporção de ascariose ocorreu na faixa etária abaixo de
Figura 1

Proporção das enteroparasitoses e do Ascaris lumbricoides por grupos estudados, RJ , 1998.

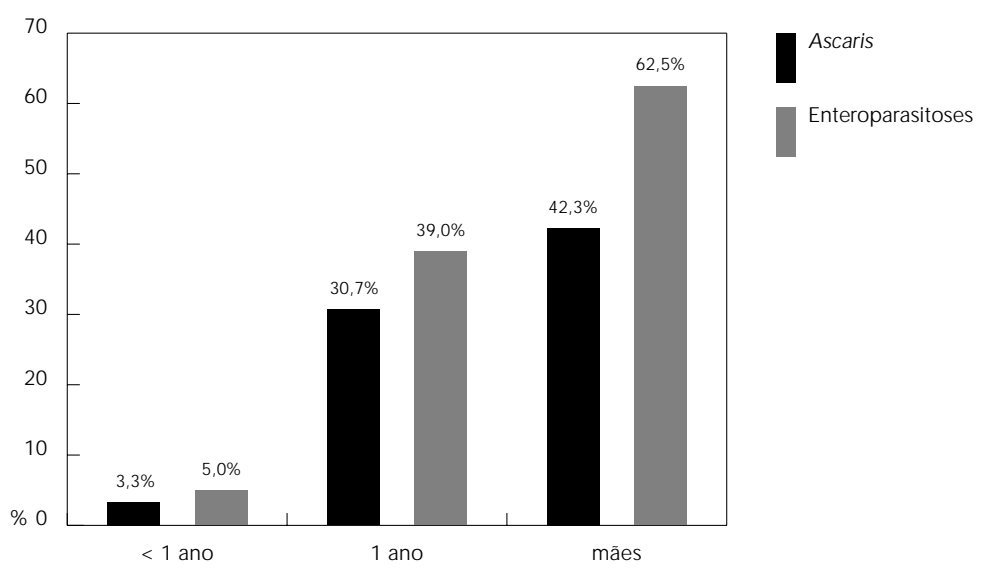

25 anos, enquanto as não infectadas eram, predominantemente, mulheres com mais de 25 anos, faixa em que a prevalência foi menos elevada (Tabela 1). A diferença observada entre estes grupos também foi estatisticamente significativa $(p=0,01)$.

A Figura 2 mostra que, na maioria dos grupos estudados, a infecção por A. lumbricoides foi considerada leve, segundo os critérios da Organização Mundial de Saúde (WHO, 1987). Entretanto, quase $38 \%$ das crianças de um ano de idade apresentaram infecção de moderada a pesada, com cargas parasitárias acima de 5.000 ovos por grama de fezes. Uma destas crianças apresentou infecção com mais de 100.000 ovos por grama de fezes (opg).

A simultaneidade do parasitismo maternoinfantil foi avaliada em 104 crianças menores de um ano e suas mães. Entre as crianças, em três casos foi diagnosticado A. lumbricoides, e em dois casos Giardia lamblia. Os resultados revelaram que as mães das três crianças parasitadas com A. Iumbricoides também apresentavam a infecção, fato que não foi observado nas duas crianças portadoras de giardiose.

\section{Discussão}

No Brasil, o parasitismo intestinal nos dois primeiros anos de vida é menos conhecido que em outras faixas etárias. Neste estudo, a presença de parasitismo foi registrada a partir do sexto mês de vida. Em três destas crianças a 
Tabela 1

Distribuição de crianças menores de 24 meses e mães de menores de um ano, por grupo de idade e prevalências do A. lumbricoides observadas. Município de Duque de Caxias, RJ . 1998.

\begin{tabular}{|c|c|c|c|c|}
\hline Variável & $\mathrm{n}$ & Casos & Prevalência (\%) & I.C. $95 \%$ \\
\hline \multicolumn{5}{|c|}{ Idade das crianças (meses)* } \\
\hline$<6$ & 56 & - & - & - \\
\hline $6-11$ & 64 & 4 & 6,3 & $2,0-16,0$ \\
\hline $12-17$ & 122 & 32 & 26,2 & $18,9-35,1$ \\
\hline $18-23$ & 145 & 51 & 35,2 & $27,5-43,6$ \\
\hline \multicolumn{5}{|c|}{ Idade das mães de menores de 1 ano (anos)** } \\
\hline$<25$ & 44 & 25 & 55,6 & $40,1-70,0$ \\
\hline $25 e+$ & 60 & 18 & 30,0 & $19,2-43,4$ \\
\hline
\end{tabular}

$* p<0,001 ; * * p=0,01$

Figura 2

Distribuição da carga parasitária do Ascaris lumbricoides por grupos estudados, RJ , 1998.

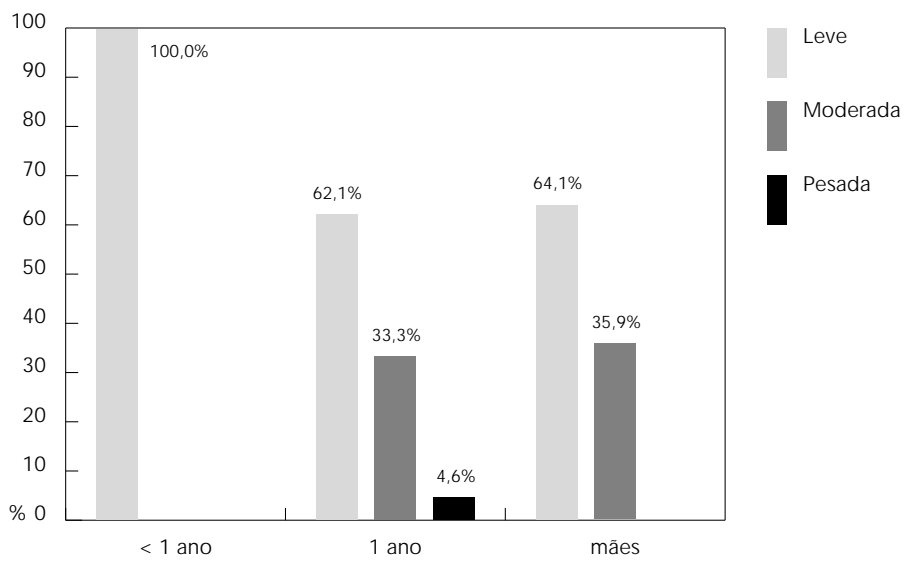

mãe também se encontrava infectada. Todavia, para o conjunto das crianças menores de um ano, a positividade encontrada para o A. Iumbricoides foi percentualmente menor, quando comparada aos resultados de outros autores (Frenzel et al., 1979; Gendrel et al., 1983; Monteiro et al., 1988; Costa-M acedo \& Rey, 1997). Para esta faixa etária, os resultados se mostraram menos precisos, devido ao tamanho reduzido da amostra examinada. A perda maior nesta faixa etária (e no grupo de mães) em relação a outra faixa estudada, deveu-se, em parte, a pouca sensibilização das mães para participarem do estudo. Por outro lado, o tipo de exame (MIF) é mais trabalhoso (coleta múltipla de fe- zes, em dias diferentes), o que gerava coletas incompletas, devolução de potes vazios e pedidos de maior prazo para coletar o material. Esses pedidos acarretavam, por sua vez, a necessidade de maior número de visitas domiciliares além do previsto (3), o que nem sempre foi possível. A perda importante nesse grupo (48\%) pode estar comprometendo as estimativas, especialmente se o grupo que não participou estiver relacionado a uma probabilidade de apresentar infecção diferente do grupo que participou.

Contudo, é importante assinalar que o parasitismo já a partir dos seis meses de vida pode estar relacionado ao período do desmame da criança, quando ocorre a introdução de novos alimentos e inicia-se uma etapa do desenvolvimento que lhe permite maior mobilidade no ambiente. Vale destacar a acentuada elevação na proporção de parasitismo nas crianças com 12-23 meses/ ano de idade, em relação às da faixa etária anterior, onde, mais uma vez, o parasitismo por Ascaris lumbricoi des configura-se como o mais freqüente nesta faixa etária. A extrema diferença encontrada entre os menores de um ano e as crianças de um ano já foi relatada por outros autores (Monteiro et al., 1988; Costa-Macedo \& Rey, 1997; Costa-Macedo et al., 1998).

Uma possível explicação para a prevalência negativa do parasitismo abaixo dos seis meses de idade pode estar relacionada à hipótese de Carlier \& Truyens (1995) que sugere o envolvimento de respostas imunológicas em filhos de mães imunes com uma proteção inicial contra a infecção por conta da transferência de anticorpos maternos contra Ascaris lumbricoides.

Como as rotas de transmissão da ascariose pressupõem contato com solo e/ ou alimentos contaminados e dependem de um tempo para 
que os ovos eliminados pelo hospedeiro no meio ambiente tornem-se infectantes (geohelmintos), surpreende-nos a maior precocidade de transmissão dessa espécie quando comparada às outras de transmissão fecal-oral direta, como os protozoários intestinais.

O parasitismo de menores de 24 meses pode (e deve) estar refletindo o elevado grau de contaminação dos ambientes peri e domiciliar nesse local, resultante das precárias condições de saneamento básico existentes. Os níveis crescentes da prevalência por idade observados nas crianças devem estar relacionados ao processo de crescimento e desenvolvimento infantil (mobilidade e interação com o ambiente) e ao maior tempo de exposição às condições ambientais.

Nas mães, ao contrário do que foi observado para as crianças, as proporções tendem a diminuir com a idade. Uma hipótese para explicar a diferença observada estaria relacionada ao nível de conhecimento sobre os mecanismos de transmissão desta parasitose, a uma melhor educação sanitária e um maior nível de escolaridade nas mulheres de faixas etárias mais elevadas.

Tendo em vista as precárias condições de saneamento da comunidade onde esse estudo foi desenvolvido, os níveis de infecção materna podem estar relacionados a uma situação ambiental que representa risco de infecção por A. Iumbricoides para toda a família. O Município de Duque de Caxias não conta com nenhum domicílio ligado a uma rede geral de tratamento de esgotos e apenas cerca de $45 \%$ das moradias estão ligadas à rede geral de abastecimento de água.

Embora a carga parasitária observada no estudo tenha correspondido predominantemente à infecção leve nos três grupos estuda- dos, constatamos que nas crianças de um ano quase 38\% apresentavam infecção de moderada a pesada. Além da maior contribuição desta faixa etária na contaminação ambiental, este é também o grupo mais exposto aos riscos da infecção, que segundo Chan (1997), pode ocorrer quando o número de parasitos no hospedeiro estiver acima de sete vermes. Como a correspondência grosseira entre o número de ovos por grama de fezes e o número de vermes é de cerca de 1.000 opg/ verme (Ferreira et al., 1991), cerca de $30 \%$ dessas crianças estariam sob risco de doença parasitária por apresentarem carga parasitária igual ou superior a 7.000 opg.

Para as crianças menores de um ano, verificamos apenas carga parasitária leve, dado consistente com os achados de Ferreira et al. (1991). Este fato, entretanto, não garante que elas estejam protegidas do desenvolvimento de doença parasitária, pois além da capacidade migratória do parasito, que pode levar a manifestações clínicas desencadeadas por apenas um verme, devemos considerar a relação de diâmetro entre o verme e a luz intestinal (Silva et al., 1997b). Obstrução intestinal em crianças menores de cinco anos (Silva et al., 1997b), alterações nutricionais (Costa-Macedo \& Rey, 1990) e obstrutivas (Rathi et al., 1981) em lactentes foram observadas em crianças com cargas parasitárias leves.

Nossos resultados mostraram que A. lumbricoides foi o principal parasito encontrado nos grupos estudados e reforçam a importância do estudo dessa parasitose na população materno-infantil. A prevalência da infecção por este parasito, especialmente nas crianças menores de um ano, poderia ser melhor explorada como um indicador das condições sócioambientais e sua relação com a saú de e qualidade de vida da população.

\section{Agradecimentos}

Seremos eternamente gratas à população de Duque de Caxias que participou deste trabalho. Agradecemos à equipe de pesquisadores de campo do Projeto de Avaliação dos Impactos do Programa de Despoluição da Baía de Guanabara sobre a Saúde e Qualidade deVida (Paisqua) pela coleta e transporte do material para exame, à Marilene Adão de Paula (UERJ) pela execução das técnicas parasitológicas e à Gisele Andréia Oliveira de Araújo pela leitura da carga parasitária. O Paisqua está sendo desenvolvido pelo Núcleo de Estudos de Saúde Coletiva da UFRJ com o financiamento da Faperj. 
Referências

BLAGG, W.; SCHLOEGEL, E. L.; MANSUR, N. S. G. \& KHOLAF, G. I., 1955. A new concentration technic for the demonstration of protozoa and helminth egss in feces. American Journal of Tropical Medicineand Hygiene, 4:23-28.

CARLIER, Y. \& TRUYENS, C., 1995. Influence of maternal infection on offspring resistence towards parasites. Parasitology Today, 11:94-99.

CHAN, M. S., 1997. The global burden of intestinal nematode infections-fifty years on. Parasitology, 109:373-387.

CHAN, M. S.; BUNDY, D. A. \& KAN, P., 1994. Genetic relatedness as a determinant of predisposition to Ascaris lumbricoides and Trichuris trichiura infection. Parasitology, 108:77-80.

CHIEFFI, P. P.; WALDMAN, E. A.; DIAS, R. M. D. S.; TORRES, D. M. A. G. V.; CHIMARA, R.; MIZUMOTO, L. C.; SILVA, A. M. A. \& UEHARA, M., 1988. Enteroparasitoses no Município de Guarulhos, SP, Brasil. 1- Prevalência de infecção entre residentes no bairro de Taboatão em junho de 1984. Revista do Instituto Adol fo Lutz, 48:75-80.

COSTA-M ACEDO, L. M . \& REY, L., 1990. Ascarislumbricoides in neonate: evidence of congenital transmission of intestinal nematodes. Revista do Instituto de Medicina Tropical de São Paulo, 32:351354.

COSTA-MACEDO, L. M. \& REY, L., 1997. Frequency and precocity of human intestinal parasitism in a group of infants from Rio de Janeiro, Brasil. Revista do Instituto de Medicina Tropical de São Paulo, 39:305-306.

COSTA-MACEDO, L. M.; MACHADO SILVA, J. R.; RODRIGUES-SILVA, R.; OLIVEIRA, L. M. \& VIANNA, M. S. R., 1998. Enteroparasitoses em pré-escolares de comunidades favelizadas da cidade do Rio de Janeiro, Brasil. Cadernos de Saúde Pública, 14:851-855.

COUTINHO, J. O., 1956. Notas sobre modificações do "MIFC" na conservação de fezes para pesquisa de cistos de protozoários. Arquivos da Faculdade de Higienee Saúde Pública, 10:65-70.

FERREIRA, C. S.; FERREIRA, M. U. \& NOGUEIRA, M. R., 1991. Prevalência e intensidade de infecção por Ascaris lumbricoides em amostra populacional urbana (São Paulo, SP). Cadernos de Saúde Pública, 7:82-89.

FRENZEL, A.; TORRES, P.; GUERRERO, S.; GESCHE, W.; MONTEFUSCO, A. \& MARIN, F., 1979. Parasitosis intestinal en lactentes y su relación con la infección de sus manipuladores de alimentos y el saneamiento ambiental. Revista Médica de Chile, 107:343-351.
GENDREL, D.; KOMBILA, M .; KHAYATI, A.; BOURDIL, M.; ENGOHAN, E. \& RICHARD- LENOBLE, D., 1983. Précocité d'apparition du parasitisme intestinal chez le nourisson en Afrique Equatoriale. Annales de Pédiatrie, 30:453-456.

GROSS, R.; SCHELL, B.; MOLINA, M. C. B.; LEÃO, M. A. C. \& STRACK, U., 1989. The impact of improvement over water suppy and sanitation facilities on diarrhea and intestinal parasites: a brazilian experience with children in two low-income urban communities. Revista de Saúde Pública, 23: 214-220.

IBGE (Instituto Brasileiro de Geografia e Estatística), 1997. Brasil em Números. Rio de Janeiro: IBGE.

KATZ, N.; CHAVES, A. \& PELLEGRINO, J., 1972. A simple device for quantitative stool thick-smear technique in schistosomiasis mansoni. Revista do Instituto de Medicina Tropical de São Paulo, 14: 397-400.

LUIZ, R. R.; COSTA, M. C. E.; COLETTY, P. E.; MACHADO, V. A.; AZEVEDO, R. S. \& ALMEIDA, L. M., 1997. Produção e análise de dados em avaliações de impacto de intervenções ambientais: perspectivas e limitações - Os inquéritos epidemiológicos. In: Saneamento e Saúde em Países em Desenvolvimento (L. Heller, L. R. S. Moraes, T. C. N. Monteiro, M. J. Sales, L. M. Almeida \& J. Câncio, org.), pp. 122-136, Rio de Janeiro: CC \& P Editores Ltda.

MONTEIRO, C. A.; CHIEFFI, P. P.; BENÍCIO, M. H. A.; DIAS, R. M. S.; TORRES, D. M. A. G. V. \& MANGINI, A. C. S., 1988. Estudo das condições de saúde das crianças do Município de São Paulo (Brasil), 1984/ 1985. VII. Parasitoses intestinais. Revista de Saúde Pública, 22:8-15.

RATHI, A. K.; BATRA, S.; CHANDRA, J. \& MEHEROTA, S. N., 1981. Ascariase causing intestinal obstrucion in a 45-day-old infant. Indian Pediatrics, 18:751-752.

SILVA, N. R.; CHAN, M. S. \& BUNDY, D. A. P., 1997a. Morbidity and mortality due to ascariasis: re-estimation and sensivity analysis of global numbers at risk. Tropical Medicine and International Health, 2:519-528.

SI LVA, N. R.; GUYATT, H. L. \& BUNDY, D. A. P., 1997b. Morbidity and mortality due to Ascaris-induced intestinal obstruction. Transactions of the Royal Society of Tropical Medicineand Hygiene, 9:31-36.

VINHA, C., 1971. Incidência, no Brasil, de helmintos transmitidos pelo solo: rotina coproscópica do ex- Departamento Nacional de Endemias Rurais. Revista Brasileira de Malariologia e Doenças Tropicais, 23:3-17.

WHO, 1987. Public health significance of intestinal parasitic infections. Bulletin of theWorld Health Organization, 65:575-588. 